\title{
Epidemiological Investigation of Canine Leishmaniasis in Southern Morocco
}

\author{
Samia Boussaa, ${ }^{1,2}$ Mohamed Kasbari, ${ }^{3}$ Amal El Mzabi, ${ }^{4}$ and Ali Boumezzough ${ }^{2}$ \\ ${ }^{1}$ Institut Supérieur des Professions Infirmières et des Techniques de Santé (ISPITS), Ministère de Santé, 40000 Marrakech, Morocco \\ ${ }^{2}$ Equipe Ecologie Animale et Environnement-Lab L2E (URAC 32), Faculté des Sciences Semlalia, Université Cadi Ayyad, \\ 40000 Marrakech, Morocco \\ ${ }^{3}$ ANSES, French Agency for Health and Safety, Animal Health Laboratory, Leishmaniasis and Sand Flies Team, \\ 94700 Maisons-Alfort, France \\ ${ }^{4}$ Equipe Modélisation Economique-Lab PEL, Faculté des Sciences Juridiques Economiques et Sociales, \\ Université Hassan 2, 20650 Mohammedia, Morocco \\ Correspondence should be addressed to Samia Boussaa; samiaboussaa@yahoo.fr
}

Received 20 April 2014; Revised 21 August 2014; Accepted 9 September 2014; Published 24 September 2014

Academic Editor: Xu-Sheng Zhang

Copyright (C) 2014 Samia Boussaa et al. This is an open access article distributed under the Creative Commons Attribution License, which permits unrestricted use, distribution, and reproduction in any medium, provided the original work is properly cited.

\begin{abstract}
Dogs are the major reservoir of Leishmania infantum, the causative agent of human and canine visceral leishmaniasis in the Mediterranean basin. In Morocco, canine leishmaniasis (CanL) is usually believed to be widespread mainly, if not only, in the northern regions and few data are available about the situation in southern parts of the country. Here, we report the results of a preliminary, clinical, and serological study carried out in 2004-2007, in four provinces of southern Morocco. Serological analyses were processed using two different Elisa techniques, a homemade Elisa test and IDVET commercial kit, and confirmed by two different western blot (WB) tests, homemade and LDBIO commercial kits. We highlighted the presence of CanL infection in southern regions, known until then as free of the disease: 19.8\% (48/243) of examined dogs displayed clinical signs compatible with CanL and the seroprevalence was particularly high, respectively, $81.8 \%$ and $87.8 \%$ by Elisa and western blot tests. Our current developed and validated homemade (Elisa and WB) tools will be cost-effective and useful for next large-scale epidemiological studies on Moroccan leishmaniasis animal reservoir.
\end{abstract}

\section{Introduction}

Canine leishmaniasis (CanL) is a zoonotic disease caused by Leishmania infantum, a trypanosomatid protozoan, transmitted through infected sand fly (Diptera: Psychodidae) bites. CanL is endemic in the Mediterranean basin, where seroprevalence ranges between ten and $37 \%[1,2]$ and symptoms in dogs occur in various combinations [3]. Both symptomatic and asymptomatic dogs are sources of the parasite and phlebotomine sand flies play an active role in the transmission of Leishmania to humans [4].

In northern Morocco, natural CanL was first reported in the region of Tangier [5]. Further subsequent cases have been reported in Taounate, Al Hoceima, Chefchaouen, and Ouezzane provinces [6], in Sefrou and Zouagha Moulay Yacoub [7], in Khemisset province [8], and in the province of Nador [9]. Leishmania infantum zymodeme MON-1 is the unique causative agent of CanL in the northern slopes of the Rif mountains [6] and L. infantum MON-24 was identified in dogs from the Pre-Rif area [10]. In the Rif region specifically, dog seroprevalence ranges between ten and $41 \%$ [9] and $L$. infantum strains are highly virulent $[8,9]$.

In northern slope of High Atlas Moroccan mountains, Dereure et al. [11] reported the presence of dogs infected with L. tropica (MON-102 and MON-113). Additionally, Guessous-Idrissi et al. [12] reported a case of dog with visceral leishmaniasis caused by L. tropica in Taounate province. More recently, in Al Hoceima province, L. tropica MON-279 was identified in dogs with clinical signs of CanL [13].

To date, only sporadic cases of human visceral leishmaniasis are reported in southern Morocco [14, 15]. In the present study we report the results of clinical and serological 
TABLE 1: Species composition of the sand fly fauna in study area.

\begin{tabular}{|c|c|c|}
\hline Province & Species composition & References \\
\hline \multirow{8}{*}{ Al Haouz } & Phlebotomus papatasi & \multirow{8}{*}[33,36,37,45]{} \\
\hline & P. sergenti & \\
\hline & P. perniciosus ${ }^{*}$ & \\
\hline & P. longicuspis & \\
\hline & P. alexandri & \\
\hline & Sergentomyia fallax & \\
\hline & S. minuta & \\
\hline & S. dreyfussi & \\
\hline \multirow{6}{*}{ Azilal } & Phlebotomus papatasi & \multirow{6}{*}[36,37,46]{} \\
\hline & P. sergenti & \\
\hline & P. perniciosus ${ }^{*}$ & \\
\hline & P. longicuspis & \\
\hline & Sergentomyia fallax & \\
\hline & S. minuta & \\
\hline \multirow{10}{*}{ Chichaoua } & Phlebotomus perniciosus ${ }^{*}$ & \multirow{10}{*}[34,36,37]{} \\
\hline & P. sergenti & \\
\hline & P. longicuspis & \\
\hline & P. ariasi & \\
\hline & P. papatasi & \\
\hline & P. alexandri & \\
\hline & P. mariae & \\
\hline & Sergentomyia fallax & \\
\hline & S. minuta & \\
\hline & S. dreyfussi & \\
\hline \multirow{5}{*}{ Marrakech } & Phlebotomus papatasi & \multirow{5}{*}[36,37,47,48]{} \\
\hline & P. sergenti & \\
\hline & P. longicuspis & \\
\hline & Sergentomyia fallax & \\
\hline & S. minuta & \\
\hline
\end{tabular}

${ }^{*}$ As atypical form [36-39].

surveys on CanL in southern Morocco, an area traditionally considered to be free from CanL in spite of the omnipresence of dogs and phlebotomine sand flies. Our investigations were carried out in four provinces of Morocco: Al Haouz, Azilal, Chichaoua, and Marrakech, where species composition of the sand fly fauna was also established (Table 1).

\section{Materials and Methods}

2.1. Sampling. An epidemiological survey was carried out in 27 localities (Figure 1) in four provinces of southern Morocco: Al Haouz $\left(31^{\circ} 22^{\prime} \mathrm{N}, 7^{\circ} 51^{\prime} \mathrm{W}\right)$, Azilal $\left(31^{\circ} 58^{\prime} \mathrm{N}\right.$, $\left.6^{\circ} 34^{\prime} \mathrm{W}\right)$, Chichaoua $\left(31^{\circ} 32^{\prime} \mathrm{N}, 8^{\circ} 45^{\prime} \mathrm{W}\right)$, and Marrakech $\left(31^{\circ} 36^{\prime} \mathrm{N}, 8^{\circ} 02^{\prime} \mathrm{W}\right)$.

Two hundred and ten dogs in 2004-2006 and 33 dogs in 2007 were studied. An individual form was completed for each dog: date, locality name and environment, dog age, sex, type (domestic or feral), and Leishmania specific clinical signs. The detail of sampling is shown in Table 2.

Because of practical, economic, and security constraints, it was not possible in 2004-2006 to take blood samples from

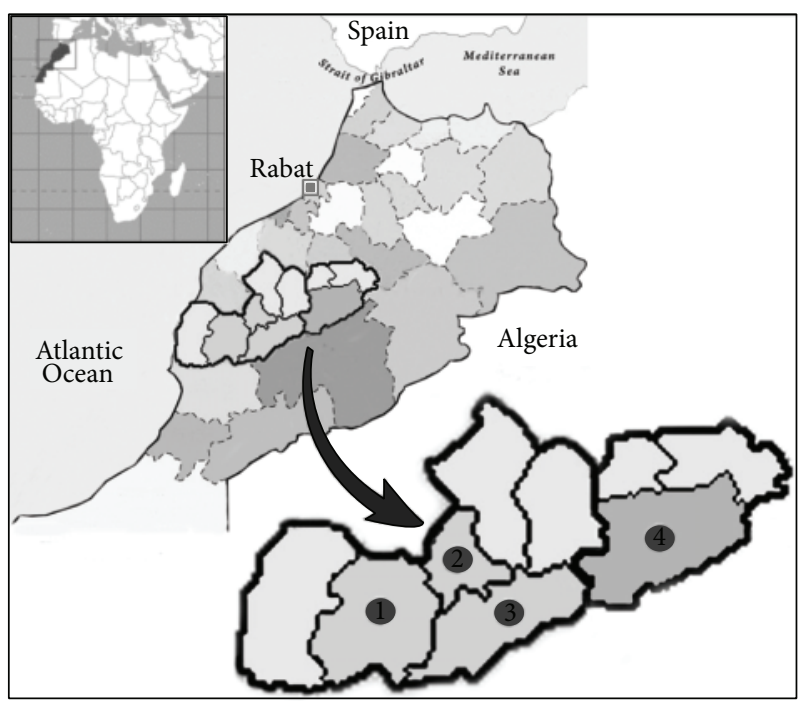

FIGURE 1: Moroccan study area provinces: (1) Chichaoua; (2) Marrakech; (3) Al Haouz; (4) Azilal.

stray and feral dogs with unknown rabies infection status. In 2007, after clinical examination, blood was collected by jugular vein puncture and 33 sera were stored at $-80^{\circ} \mathrm{C}$ for serological tests.

2.2. Serological Assays. For serological analyses, the 33 sera were processed using two ELISA techniques: a homemade ELISA test and IDVET commercial kit and the results were confirmed by two western blot (homemade and LDBIO commercial kit) techniques.

2.2.1. Preparation of the L. Infantum Antigen. Antigens were produced from Leishmania infantum promastigotes grown in RPMI 1640 medium supplemented with $10 \%$ fetal calf serum (Sigma), L-glutamine (Gibco), and penicillin-streptomycin (Gibco). Log phase promastigotes were harvested by centrifugation $\left(14000 \mathrm{~g}\right.$ for $15 \mathrm{~min}$ at $\left.4^{\circ} \mathrm{C}\right)$ and washed three times in sterile phosphate buffered saline (PBS). Cells were counted and adjusted to a concentration of $3.5 \times 10^{8}$ promastigotes $/ \mathrm{mL}$ and, as previously described [16], and lysed by boiling for $5 \mathrm{~min}$ in sample buffer $(0.5 \mathrm{M}$ Tris- $\mathrm{HCl}$, pH 6.8, 0.01 M EDTA, 5\% sodium dodecyl sulphate (SDS), $5 \%$ 2-mercaptoethanol, and $0.0125 \%$ bromophenol blue). Protein concentration $(1.5 \mathrm{mg} / \mathrm{mL})$ was determined by the bicinchoninic acid method (Pierce) and antigen was stored at $-80^{\circ} \mathrm{C}$.

\subsubsection{WB Analysis}

Homemade Western Blot Kit. SDS-polyacrylamide gel electrophoresis was performed on a 15\% polyacrylamide gel with a Mini-Protean II apparatus (Biorad) and $200 \mu \mathrm{g}$ of antigen was used per gel width with molecular mass proteins standards (standard low range, BioRad) as previously described [17]. Gels were run at $50 \mathrm{~V}$ for 3 hours at room temperature. 


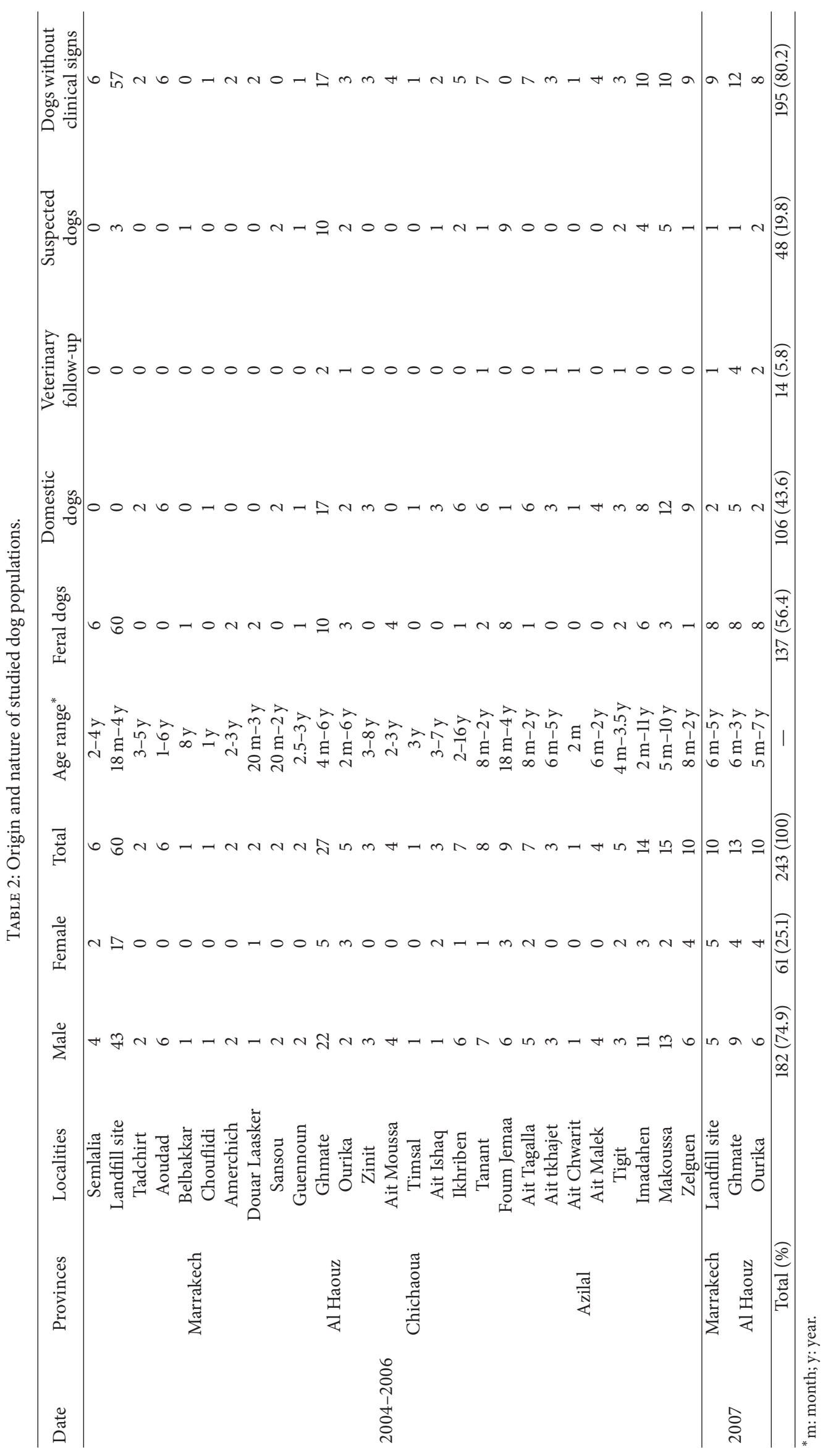


After electrophoresis, the proteins were transferred onto a nitrocellulose sheet (Hybond ECL, Amersham Biosciences). The sheets were blocked in PBS containing 5\% skimmed milk and $0.2 \%$ Tween 20 , overnight at $4^{\circ} \mathrm{C}$, and then washed five times. The dry membranes were cut and kept refrigerated.

For immunoblotting, two dilutions of sera in PBS, $0.5 \%$ skimmed milk and $0.2 \%$ Tween 20 , were used with total antigen $1 / 25$ and $1 / 100$. The nitrocellulose individual strips were incubated for 3 hours with sera, washed five times in PBS $0.2 \%$ Tween 20, and incubated in a $1 / 300$ dilution of alkaline phosphatase-conjugated rabbit anti-canine IgG (PARIS Anticorps, compiègne, France) for 2 hours. After five washes, the bound proteins were revealed with nitro blue tetrazolium (NBT) and bromo-chloro-indolyl phosphate (BCIP) as substrates. The reaction was stopped with distilled water. Positive and negative controls were included in each procedure. The interpretation was based on the presence of the antibodies to 14 and $16 \mathrm{kDa}$ antigens, which are common to all Leishmania species [18].

Commercial Western Blot Kit. Leishmania LDBIO kit (ref. LES-WB12G, Lyon, France) was used according to the manufacturer's specifications.

\subsubsection{Enzyme-Linked Immunosorbent Assay (ELISA)}

Homemade ELISA Kit. Quantification of antibodies by ELISA was performed according to following procedure adapted from Mary et al. and Correia da Costa et al. [17, 19].

96-well microtiter plates were coated with the previously described antigen diluted at four different concentrations (5, 10,20 , and $50 \mu \mathrm{g} / \mathrm{mL}$ ) in PBS, $\mathrm{pH} 7.2$ in a volume of $100 \mu \mathrm{L}$. Plates were then incubated for 2 hours at $37^{\circ} \mathrm{C}$ and overnight at $4^{\circ} \mathrm{C}$.

Sera were serially diluted from $1 / 40$ to $1 / 1280$ in PBS, $0.5 \%$ skimmed milk, and $0.2 \%$ Tween 20 (PBS-T) and incubated on microplates previously coated for 1 hour at $37^{\circ} \mathrm{C}$. After three washes, a labelled anti-dog IgG alkaline phosphatase conjugate (PARIS Anticorps, compiègne, France), diluted to 1/1000 in PBS-T, was added per well and incubated for 1 hour at $37^{\circ} \mathrm{C}$. Enzymatic activity was revealed by hydrolysis of para-nitrophenyl phosphate for $15 \mathrm{~min}$ at $37^{\circ} \mathrm{C}$ and the reaction stopped by adding $\mathrm{K}_{2} \mathrm{HPO}_{4} 2 \mathrm{M}$. Plates were read at $405 \mathrm{~nm}$ in an ELISA reader (Multiscan ThermoFischer).

Commercial ELISA Kit. The ID Screen Leishmaniasis Indirect (ref. LEISHS-2P, IDVET, Montpellier, France) kit was used according to the manufacturer's specifications.

2.3. Statistical Analysis. Data was analysed by crosstab and logistic regression.

\section{Results}

3.1. Characterisation of the Dog Population. A total of 243 dogs were clinically examined: $74.9 \%$ males and $43.6 \%$ domestic type (pets) versus $56.4 \%$ stray dogs. Only $5.8 \%$ dogs

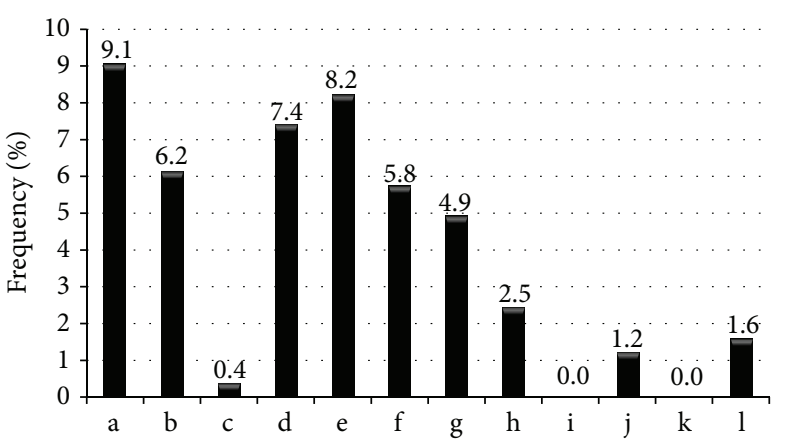

FIGURE 2: Frequency of each clinical sign displayed by all symptomatic and suspected dogs: (a) looking old; (b) cachexia; (c) anorexia; (d) weakening; (e) dry exfoliative dermatitis and/or periorbital alopecia; (f) ulcers; (g) onychogryphosis; (h) noise at walk; (i) epistaxis; (j) ocular signs (keratoconjunctivitis and uveitis); (k) lymphadenopathy; (l) splenomegaly.

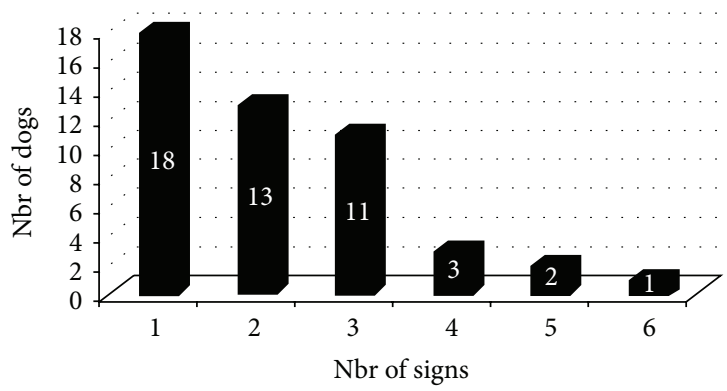

FIGURE 3: Number (Nbr) of clinical signs displayed by all symptomatic and suspected dogs.

were under veterinary follow-up and $19.8 \%$ were clinically suspected (Table 2).

In this study, we investigated twelve commonly described clinical cutaneous and visceral CanL signs, as reported in the scientific literature. Gállego [20] confirmed that the most frequent signs are skin abnormalities (dry exfoliative dermatitis, ulcers, periorbital alopecia, and onychogryphosis), but ocular signs (keratoconjunctivitis and uveitis) and lymphadenomegaly are also common. According to Solano-Gallego and Baneth [21], the main clinical signs associated with CanL are skin lesions, lymphadenomegaly, splenomegaly, ocular abnormalities, onychogryphosis, weight loss, and general poor body condition. Carré et al. [22] classified these symptoms to general, visceral, and mucocutaneous signs.

In our results, alteration of general aspect (9.1\%) and skin abnormalities (8.2\%) were the most common clinical signs, while ocular (1.2\%) and visceral (1.6\%) signs were very discrete (Figure 2).

Figure 3 shows the association of clinical signs. Most of the infected dogs were oligosymptomatic: $37.5 \%$ of symptomatic dogs presented only one sign, $27.1 \%$ two signs, $22.9 \%$ three signs, and $12.5 \%$ four or more clinical signs.

3.2. Serological Results. Firstly, concerning the validation of the homemade western blot protocol, better intensity and 
TABLE 3: Seroprevalence of canine leishmaniasis infection in southern Morocco: comparison of commercial and homemade ELISA and western blot tests.

\begin{tabular}{|c|c|c|c|c|c|}
\hline \multirow{3}{*}{ Localities } & \multirow{3}{*}{$\begin{array}{l}\text { Dogs clinically } \\
\text { suspected (\%) }\end{array}$} & \multicolumn{4}{|c|}{ Seropositive dogs (\%) } \\
\hline & & \multicolumn{2}{|c|}{ ELISA } & \multicolumn{2}{|c|}{ Western blot } \\
\hline & & Homemade kit & IDVET kit & Homemade kit & LDBIO kit \\
\hline Landfill site & $1 / 10(10)$ & $70(7 / 10)$ & $(5 / 10) 50$ & $80(8 / 10)$ & $80(8 / 10)$ \\
\hline Ghmate & $1 / 13(7.7)$ & $92.3(12 / 13)$ & (7/13) 53.8 & $92.3(12 / 13)$ & $100(13 / 13)$ \\
\hline Ourika & $2 / 10(20)$ & $80(8 / 10)$ & $(2 / 10) 20$ & $60(6 / 10)$ & $80(8 / 10)$ \\
\hline Total & $4 / 33(12.1)$ & $81.8(27 / 33)$ & $42.4(14 / 33)$ & $78.8(26 / 33)$ & $87.8(29 / 33)$ \\
\hline 95\% confidence interval & {$[0.97 ; 23.2]$} & {$[68.6 ; 94.9]$} & {$[25.5 ; 59.2]$} & {$[64.8 ; 92.7]$} & {$[82.1 ; 93.5]$} \\
\hline
\end{tabular}

TABLE 4: Concordance percentage between the different serological kits.

\begin{tabular}{lccc}
\hline & & \multicolumn{2}{c}{ ELISA } \\
& & Homemade & IDVET \\
\hline \multirow{2}{*}{ WB } & Homemade & $88 \%$ & $60.6 \%$ \\
& LDBIO & $94 \%$ & $54.6 \%$ \\
\hline ELISA & Homemade versus IDVET & $60.6 \%$ \\
WB & Homemade versus LDBIO & $88 \%$ \\
\hline
\end{tabular}

readability of antigenic bands were found with sera diluted to $1 / 100$ in PBS rather than dilution of $1 / 25$.

Besides, homemade ELISA protocol showed better results when antigen coating concentration was $20 \mu \mathrm{g} / \mathrm{mL}$ in a volume of $100 \mu \mathrm{L}$ per well $(2 \mu \mathrm{g} /$ well $)$. For the next broader epidemiological studies on prevalence of canine leishmaniasis in all these preprospected areas, we recommend the use of a sera dilution of 1/100. A threshold at $0.24 \mathrm{OD}$ was established after testing 30 negative control sera (mean +2 $\mathrm{SD})$.

The highest seroprevalence of leishmaniasis infection was found to be, respectively, $81.8 \%$ (95\% confidence interval (CI): 68.6-94.9) and $87.8 \%$ (95\% CI: 82.1-93.5), with the homemade ELISA test and LDBIO commercial western blot kit (Table 3).

With the exception of IDVET commercial ELISA kit, all tests revealed a very high prevalence of infection, around $80 \%$. No significant difference (Fisher's exact test, $P>$ 0.5) was found between western blot and ELISA results, probably because of high titer of anti-Leishmania antibodies within this population of dogs. Among the 29 seropositive dogs, only four were symptomatic (13.8\%). As expected, all clinically suspected dogs were both ELISA and western blot seropositive.

The best concordance percentage (94\%) was found between LDBIO commercial western blot kit and homemade ELISA test (Table 4). Only two samples out of 33 were negative with both ELISA tests but positive with the western blot kits. The concordance percent between the commercial IDVET and homemade ELISA tests was low (60.6\%) where the homemade ELISA test was much more sensitive. However, the significant difference (Fisher's exact test, $P=0.002$ ) in sensitivity between the two ELISA techniques may be due to the conservation of sera samples. The IDVET ELISA kit was the last test used when sera had already undergone several cycles of freeze-thawing. We also cannot exclude a problem of batch or inappropriate conservation of IDVET kit reagents themselves.

Western blot was the most sensitive test and there was no signification difference (Fisher's exact test, $P>0.5$ ) between the sensitivity of the commercial versus homemade western blot kits.

Using a crosstab analysis, the results of the Chi-square test showed no significant correlation between clinical signs of disease and seropositivity $(P>0.05$ and Cramer's $V<0.70)$. Logistic regression was applied to study further possible correlations between the disease, on the one hand, and the locality, age, sex, and type of dogs (domestic or feral), on the other hand. Our results based on $P$ value and Cox and Snell's and Nagelkerke's $R$-square measures showed no significant correlation between disease and locality ( $\mathrm{Sig}=0.205)$, age ( $\mathrm{Sig}=$ $0.058)$, sex (Sig = 0.109), or type of dogs ( $\operatorname{Sig}=0.999)$.

\section{Discussion}

CanL constitutes a considerable veterinary challenge, as well as important public health problem, because infected dogs, ill or asymptomatic, act as reservoir hosts for the human disease: sand flies infected by a blood meal taken from infected dogs may, during a later blood meal, infect humans.

With the exception of sporadic cases of human visceral leishmaniasis $[14,15]$, southern Morocco is currently considered L. infantum- and CanL-free area. To provide a better understanding of $L$. infantum epidemiology in southern regions of Morocco, clinical and serological surveys were carried out to establish the presence of L. infantum in canine populations.

The western blot analysis is a valuable tool for epidemiological studies on visceral leishmaniasis especially when it is associated, for confirmation, with Elisa test. It provides a significant increase in the sensitivity, because the $14-\mathrm{kD}$ and/or 16-kD antigens are recognized by most part of infected animals or patients (if not all) and show no cross-reactivity $[17,23]$.

We showed the presence of L. infantum in our study area with a high serological prevalence in dogs, around $80 \%$. Therefore, southern Morocco might be highly endemic compared to the lower range seroprevalence (10 to $40 \%$ ) usually reported in northern Morocco $[8,9]$ and in the Mediterranean area [24-26]. 
These unusual high seroprevalence results could be explained by different factors. Firstly, the previous multiple and successive stray dog culling campaigns have been more frequent and regular in the northern populated provinces, well known for leishmaniasis endemicity, rather than in south areas. Secondly, the previous leishmaniasis seroprevalence studies, carried out in the northern Morocco or in European countries around Mediterranean basin, concerned rather pet dogs with veterinary follow-up, while 56.4\% (137/243) of animals in our study were stray dogs (Table 2), and among the $43.6 \%$ of domestic dogs with owners (pets), only $5.8 \%$ have been subjected to veterinary follow-up and possible treatment. Lastly, considering the small sampling size of this preliminary study, an unknown selection bias cannot be completely excluded.

Our results corroborate with Dereure et al. [27] who demonstrated the presence of an autochthonous canine visceral leishmaniasis cycle in the High and Anti-Atlas mountains of Morocco, not far from our study area.

Clinical diagnosis of leishmaniasis can be difficult due to the great variety of symptoms and should, therefore, be confirmed by parasitological, serological, or molecular methods [20]. We showed that all clinically suspected dogs (4/33) were Elisa and western blot seropositive. However, CanL usually shows a high prevalence of subclinical infection $[21,22]$, commonly explained by the long incubation time of this disease [28] and here supported by the high frequency of asymptomatic dogs (86.2\%) we found.

Besides, few human visceral leishmaniasis cases reported in our study area suggest that the dynamic of these southern Moroccan foci of CanL is different from northern ones. This unusual pattern has sometimes been observed in Khemisset province (northern Morocco) where CanL does not sustain human visceral leishmaniasis [29]. CanL and human visceral leishmaniasis distribution can geographically overlap where the incidence of the former is much higher [30].

On the other hand, the distributions of L. infantum and L. tropica overlap in Morocco. The causative agents of cutaneous leishmaniasis in Sefrou province (northern Morocco) are attributed to both $L$. tropica and L. infantum by species-specific ITS1-PCR-RFLP assay [31]. Furthermore, several cases of canine visceral leishmaniasis caused by $L$. tropica have been reported in regions where canine visceral leishmaniasis is caused by L. infantum [32].

In our study area, Azilal, Chichaoua, and El Haouz provinces are simultaneously anthroponotic foci of $L$. tropica cutaneous leishmaniasis [33-35]. Previously, some zymodemes of $L$. tropica have been several times found in dogs in anthroponotic foci of L. tropica cutaneous leishmaniasis in Taounate and Al Hoceima northern provinces [12,13] but also in south part (Azilal province) [11]. Consequently, in our study area, we cannot exclude the possibility of visceral leishmaniasis in dogs that could be due to some specific strains of L. tropica. Therefore, this study should be continued by the isolation and typing of the strains circulating in all southern CanL foci.

Moreover, concerning the vector, L. infantum in Morocco is known to be transmitted by two species of the subgenus
Larroussius, Phlebotomus perniciosus and P. ariasi [15]. However, our previous entomological investigations in this area (Table 1) showed the presence of three species of subgenus Larroussius: P. ariasi, atypical morph of P. perniciosus [36-39], and $P$. longicuspis.

The vectorial role of $P$. ariasi has been shown in northern Morocco [40], while the epidemiological roles of atypical morph of $P$. perniciosus and $P$. longicuspis are not yet elucidated. Furthermore, P. papatasi and some species of the genus Sergentomyia, largely spread throughout Morocco and our study area, might also be able to transmit $L$. infantum $[41,42]$. Hence, it is essential to identify the still unknown sand fly species involved in the CanL transmission in these southern regions. It could help to understand the reason of low prevalence of human visceral leishmaniasis in these southern CanL foci.

In southern Algeria, human visceral leishmaniasis is known to be sporadic but during the last decade the number of cases has increased significantly whereas entomological investigations showed the absence of sand flies of subgenus Larroussius [43].

Regarding canine leishmaniasis control, it remains a difficult task in Morocco because of important stray and feral dog populations, low attendance level to veterinary clinics by the owners of domestic dogs, rare use of insecticideimpregnated dog collars, an absence of proven effective vaccines at affordable cost and chemotherapeutic agents having a limited efficacy for carrier dogs, and a high cost.

For control, the World Health Organization [44] recommends the treatment of human cases, insecticide vector control, and infected dog sacrifice for the control of canine visceral leishmaniasis. So, the elimination of infected or at least symptomatic stray and feral dogs is justified in Morocco. It is an operation integrated into a global strategy of control of canine zoonosis with high impact on public health (especially rabies, echinococcosis, and leishmaniasis), but it is an activity difficult to realize. This suggests the need for a strong collaboration between the veterinary and medical services, the authorities, and the local populations.

To conclude, we reported a high seroprevalence of CanL in southern Morocco, although it is considered, until now, to be free of this infection. Both of our homemade serological kits were validated, are cost-effective, and will be useful for further large-scale epidemiological studies on the animal reservoir of visceral leishmaniasis.

These preliminary results could be a helpful basis for further investigations in these CanL high prevalent foci of southern regions in order to identify the still unknown sand fly species involved in the transmission, to isolate and characterize the circulating Leishmania strains, and to understand better the reason of lower prevalence of human visceral leishmaniasis in the southern Morocco.

\section{Conflict of Interests}

The authors declare that they have no conflict of interests regarding the publication of this paper. 


\section{Acknowledgments}

This modest work is dedicated to the memory of Khalil Ramaoui, the authors' laboratory colleague who died during a field mission. The authors are very grateful to Bernard Pesson and Shazia Mahamdallie for the critical review of the paper and kind assistance.

\section{References}

[1] R. Fisa, M. Gállego, S. Castillejo et al., "Epidemiology of canine leishmaniosis in Catalonia (Spain): the example of the Priorat focus," Veterinary Parasitology, vol. 83, no. 2, pp. 87-97, 1999.

[2] V. Sideris, G. Papadopoulou, E. Dotsika, and E. Karagouni, "Asymptomatic canine leishmaniasis in Greater Athens area, Greece," European Journal of Epidemiology, vol. 15, no. 3, pp. 271-276, 1999.

[3] V. J. Kontos and A. E. Koutinas, "Old world canine leishmaniasis," Compendium on Continuing Education for the Practising, vol. 15, pp. 949-960, 1993.

[4] R. Molina, C. Amela, J. Nieto et al., "Infectivity of dogs naturally infected with Leishmania infantum to colonized Phlebotomus perniciosus," Transactions of the Royal Society of Tropical Medicine and Hygiene, vol. 88, no. 4, pp. 491-493, 1994.

[5] G. Jeaume, "Un cas de leishmaniose naturelle généralisée chez le chien au Maroc," Bulletin de la Société de Pathologie Exotique, vol. 25, pp. 225-227, 1932.

[6] R. Nejjar, M. Lemrani, A. Malki, S. Ibrahimy, H. Amarouch, and A. Benslimane, "Canine leishmaniasis due to Leishmania infantum MON-1 in northern Morocco," Parasite, vol. 5, no. 4, pp. 325-330, 1998.

[7] R. Nejjar, M. Lemrani, L. Boucedda, H. Amarouch, and A. Benslimane, "Variation in antibody titres against Leishmania infantum in naturally infected dogs in northern Morocco," Revue de Medecine Veterinaire, vol. 151, no. 8-9, pp. 841-846, 2000.

[8] A. Natami, H. Sahibi, S. Lasri, M. Boudouma, N. GuessoussIdrrissi, and A. Rhalem, "Serological, clinical and histopathological changes in naturally infected dogs with Leishmania infantum in the khemisset province, Morocco," Veterinary Research, vol. 31, no. 3, pp. 355-363, 2000.

[9] M. Rami, T. Atarhouch, M. Sabri, M. Cadi Soussi, T. Benazzou, and A. Dakkak, "Canine leishmaniasis in the Rif mountains (Moroccan Mediterranean Coast): sero-epidemiological survey," Parasite, vol. 10, no. 1, pp. 79-85, 2003.

[10] C. Haralambous, A. Dakkak, F. Pratlong, J.-P. Dedet, and K. Soteriadou, "First detection and genetic typing of Leishmania infantum MON-24 in a dog from the Moroccan Mediterranean coast: genetic diversity of MON-24," Acta Tropica, vol. 103, no. 1, pp. 69-79, 2007.

[11] J. Dereure, J.-A. Rioux, M. Gallego, J. Perieres, F. Pratlong, and J. H. Mahjour Saddiki, "Leishmania tropica in Morocco: Infection in dogs," Transactions of the Royal Society of Tropical Medicine and Hygiene, vol. 85, no. 5, p. 595, 1991.

[12] N. Guessous-Idrissi, B. Berrag, M. Riyad, H. Sahibi, M. Bichichi, and A. Rhalem, "Short report: Leishmania tropica: etiologic agent of a case of canine visceral leishmaniasis in Northern Morocco," The American Journal of Tropical Medicine and Hygiene, vol. 57, no. 2, pp. 172-173, 1997.
[13] M. Lemrani, R. Nejjar, and F. Pratlong, "A new Leishmania tropica zymodeme-causative agent of canine visceral leishmaniasis in Northern Morocco," Annals of Tropical Medicine and Parasitology, vol. 96, no. 6, pp. 637-638, 2002.

[14] Moroccan Ministry of Health, "Lutte Contre les Leishmanioses. Direction de L'épidémiologie et de Lutte Contre les Maladies. Service des Maladies Parasitaires," in Guide des Activités, 2010.

[15] M. Rhajaoui, "Human leishmaniases in Morocco: a nosogeographical diversity," Pathologie Biologie, vol. 59, no. 4, pp. 226229, 2011.

[16] M. J. Aisa, S. Castillejo, M. Gallego et al., "Diagnostic potential of Western blot analysis of sera from dogs with leishmaniasis in endemic areas and significance of the pattern," The American Journal of Tropical Medicine and Hygiene, vol. 58, no. 2, pp. 154159, 1998.

[17] C. Mary, D. Lamouroux, S. Dunan, and M. Quilici, "Western blot analysis of antibodies to Leishmania infantum antigens: potential of the $14-\mathrm{kD}$ and $16-\mathrm{kD}$ antigens for diagnosis and epidemiologic purposes," The American Journal of Tropical Medicine and Hygiene, vol. 47, no. 6, pp. 764-771, 1992.

[18] O. Aoun, C. Mary, C. Roqueplo et al., "Canine leishmaniasis in South-East of France: screening of Leishmania infantum antibodies (Western blotting, ELISA) and parasitaemia levels by PCR quantification," Veterinary Parasitology, vol. 166, no. 1-2, pp. 27-31, 2009.

[19] J. M. Correia da Costa, A. B. Neogy, I. Vouldoukis, M. L. Sampaio Silva, M. Gentilini, and L. Monjour, "Antigenic components of partially purified antigens of Leishmania donovani infantum recognized by sera from dogs with asymptomatic or active visceral leishmaniasis," The American Journal of Tropical Medicine and Hygiene, vol. 55, no. 5, pp. 511-515, 1996.

[20] M. Gállego, “Zoonosis emergentes por patógenos parásitos: las leishmaniosis," Revue Scientifique et Technique (International Office of Epizootics), vol. 23, pp. 661-676, 2004.

[21] L. Solano-Gallego and G. Baneth, "Canine leishmaniosis-a challenging zoonosis," European Journal of Companion Animal Practice, vol. 18, pp. 232-241, 2008.

[22] N. Carré, M. Collot, P. Guillard, M. Horellou, and J.-P. Gangneux, "Visceral leishmaniasis epidemiology, diagnosis, treatment and prophylaxis," Journal de Pharmacie Clinique, vol. 29, no. 3, pp. 121-148, 2010.

[23] P. Marty, A. Lelievre, J. F. Quaranta, A. Rahal, M. GariToussaint, and Y. le Fichoux, "Use of the leishmanin skin test and Western blot analysis for epidemiological studies in visceral leishmaniasis areas: experience in a highly endemic focus in Alpes-Maritimes (France)," Transactions of the Royal Society of Tropical Medicine and Hygiene, vol. 88, no. 6, pp. 658-659, 1994.

[24] J. Dedet, F. B. Osman, A. Chadli, H. Croset, and J. A. Rioux, "Leishmaniasis in Tunisia. Sero immunological survey of the frequency of infestation," Annales de Parasitologie Humaine et Comparee, vol. 48, no. 5, pp. 653-660, 1973.

[25] S. Mansueto, M. D. Miceli, and P. Quartararo, "Counterimmunoelectrophoresis (CIEP) and ELISA tests in the diagnosis of canine leishmaniasis," Annals of Tropical Medicine and Parasitology, vol. 76, no. 2, pp. 229-231, 1982.

[26] I. Amusategui, A. Sainz, E. Aguirre, and M. A. Tesouro, "Seroprevalence of Leishmania infantum in Northwestern Spain, an area traditionally considered free of leishmaniasis," Annals of the New York Academy of Sciences, vol. 1026, pp. 154-157, 2004.

[27] J. Dereure, I. D. Velez, F. Pratlong et al., "La leishmaniose viscérale autochtone au Maroc méridional. Présence 
de Leishmania infantum MON-1 chez le Chien en zone présaharienne," in Leishmania. Taxonomie et Phylogenèse. Applications éco-épidémiologiques, pp. 421-425, Colloque International CNRS/INSERM, Montpellier, France, 1986.

[28] R. W. Ashford and S. Bettini, "In the leishmaniasis in Biology and Medicine," in Ecology and Epidemiology, W. Peters and R. Killick-Kendrick, Eds., New World, Academic Press, London, UK, 1987.

[29] H. Sahibi, A. Natami, A. Moufaid, S. Lasri, and A. Rhalem, "Clinical and seroepidemiological study of Leishmaniasis in Northern Morocco," Revue Marocaine des Sciences Agronomiques et Vétérinaires, vol. 21, no. 4, pp. 227-232, 2001.

[30] M. Gramiccia, "Recent advances in leishmaniosis in pet animals: epidemiology, diagnostics and anti-vectorial prophylaxis," Veterinary Parasitology, vol. 181, no. 1, pp. 23-30, 2011.

[31] H. Asmae, A. Fatima, F. Hajiba et al., "Coexistence of Leishmania tropica and Leishmania infantum in Sefrou province, Morocco," Acta Tropica, vol. 130, no. 1, pp. 94-99, 2014.

[32] M. Rhajaoui, A. Nasereddin, H. Fellah et al., "New clinicoepidemiologic profile of cutaneous leishmaniasis, Morocco," Emerging Infectious Diseases, vol. 13, no. 9, pp. 1358-1360, 2007.

[33] K. Ramaoui, S. Guernaoui, and A. Boumezzough, "Entomological and epidemiological study of a new focus of cutaneous leishmaniasis in Morocco," Parasitology Research, vol. 103, no. 4, pp. 859-863, 2008.

[34] S. Guernaoui, A. Boumezzough, B. Pesson, and G. Pichon, "Entomological investigations in Chichaoua: an emerging epidemic focus of cutaneous leishmaniasis in Morocco," Journal of Medical Entomology, vol. 42, no. 4, pp. 697-701, 2005.

[35] F. Pratlong, J. A. Rioux, and J. Dereure, "Leishmania tropica au Maroc. IV. Diversité isozymique intrafocale," Ann Parasitol Hum Comp journal, vol. 66, pp. 100-104, 1991.

[36] S. Guernaoui, B. Pesson, A. Boumezzough, and G. Pichon, "Distribution of phlebotomine sandflies, of the subgenus Larroussius, in Morocco," Medical and Veterinary Entomology, vol. 19, no. 1, pp. 111-115, 2005.

[37] S. Boussaa, A. Boumezzough, P. E. Remy, N. Glasser, and B. Pesson, "Morphological and isoenzymatic differentiation of Phlebotomus perniciosus and Phlebotomus longicuspis (Diptera: Psychodidae) in Southern Morocco," Acta Tropica, vol. 106, no. 3, pp. 184-189, 2008.

[38] I. Benabdennbi, B. Pesson, M. Cadi-Soussi, and F. M. Marquez, "Morphological and isoenzymatic differentiation of sympatric populations of Phlebotomus perniciosus and Phlebotomus longicuspis (Diptera: Psychodidae) in northern Morocco," Journal of Medical Entomology, vol. 36, no. 1, pp. 116-120, 1999.

[39] B. Pesson, J. S. Ready, I. Benabdennbi et al., "Sandflies of the Phlebotomus perniciosus complex: mitochondrial introgression and a new sibling species of $P$. longicuspis in the Moroccan Rif," Medical and Veterinary Entomology, vol. 18, no. 1, pp. 25-37, 2004.

[40] A. Hamdani, Etude de la faune phlébotomienne dans trois foyers de leishmanioses au Nord du Maroc: espèces, abondance, saisonnalité et incrimination du vecteur. Thèse de 3ème cycle [Ph.D. thesis], Faculté des Sciences Semlalia, Marrakech, Morocco, 1999.

[41] R. Yavar, K. Hadi, A. M. Reza et al., "First detection of Leishmania infantum DNA in wild caught Phlebotomus papatasi in endemic focus of cutaneous leishmaniasis, South of Iran," Asian Pacific Journal of Tropical Biomedicine, vol. 3, no. 10, pp. 825-829, 2013.
[42] M. W. Senghor, M. N. Faye, B. Faye et al., "Ecology of phlebotomine sand flies in the rural community of Mont Rolland (Thiès region, Senegal): area of transmission of canine leishmaniasis," PLoS ONE, vol. 6, no. 3, Article ID e14773, 2011.

[43] K. Benallal, B. Gassen, L. Bouiba, J. Depaquit, and Z. Harrat, "Entomological investigation following the resurgence of human visceral leishmaniasis in southern Algeria," Acta Tropica, vol. 128, no. 3, pp. 518-521, 2013.

[44] WHO, "Control of the leishmaniasis: report of a meeting of the WHO expert committee on the control of Leishmaniases," Tech. Rep. no. 949, World Health Organization, Geneva, Switzerland, 2010.

[45] S. Boussaa, B. Pesson, and A. Boumezzough, "Faunistic study of the sandflies (diptera: Psychodidae) in an emerging focus of cutaneous leishmaniasis in Al Haouz province, Morocco," Annals of Tropical Medicine and Parasitology, vol. 103, no. 1, pp. 73-83, 2009.

[46] S. Boussaa, M. Neffa, B. Pesson, and A. Boumezzough, "Phlebotomine sandflies (Diptera: Psychodidae) of southern Morocco: results of entomological surveys along the Marrakech-Ouarzazat and Marrakech-Azilal roads," Annals of Tropical Medicine and Parasitology, vol. 104, no. 2, pp. 163-170, 2010.

[47] S. Boussaa, S. Guernaoui, B. Pesson, and A. Boumezzough, "Seasonal fluctuations of phlebotomine sand fly populations (Diptera: Psychodidae) in the urban area of Marrakech, Morocco," Acta Tropica, vol. 95, no. 2, pp. 86-91, 2005.

[48] S. Boussaa, B. Pesson, and A. Boumezzough, "Phlebotomine sandflies (Diptera: Psychodidae) of Marrakech city, Morocco," Annals of Tropical Medicine and Parasitology, vol. 101, no. 8, pp. 715-724, 2007. 


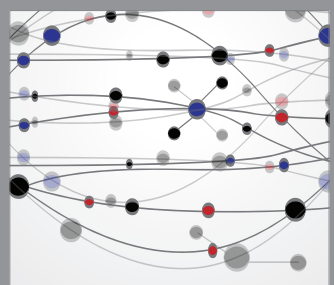

The Scientific World Journal
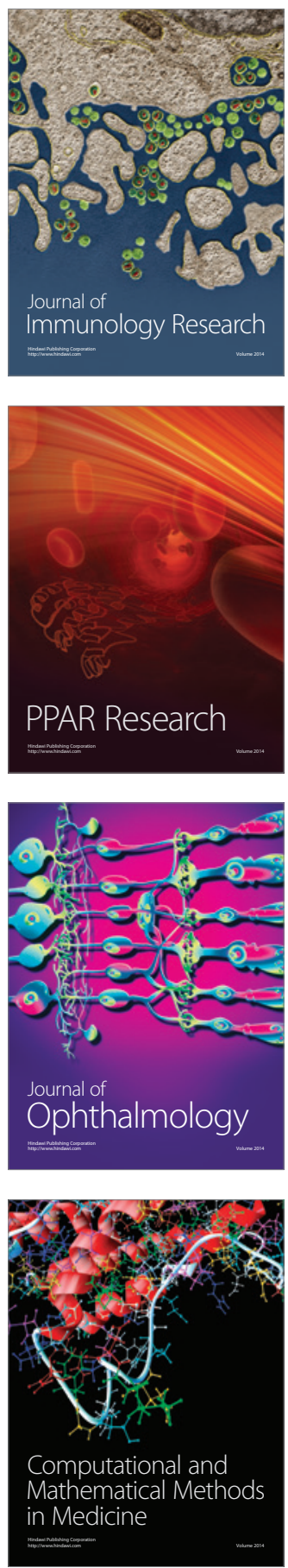

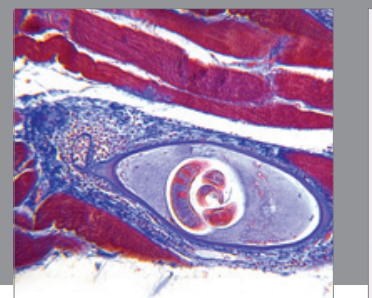

Gastroenterology

Research and Practice
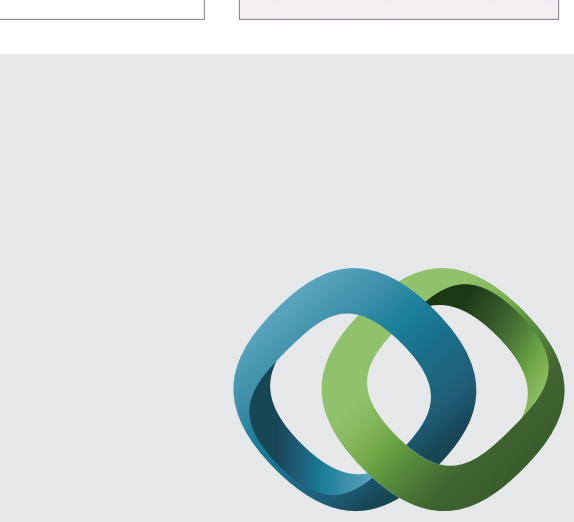

\section{Hindawi}

Submit your manuscripts at

http://www.hindawi.com
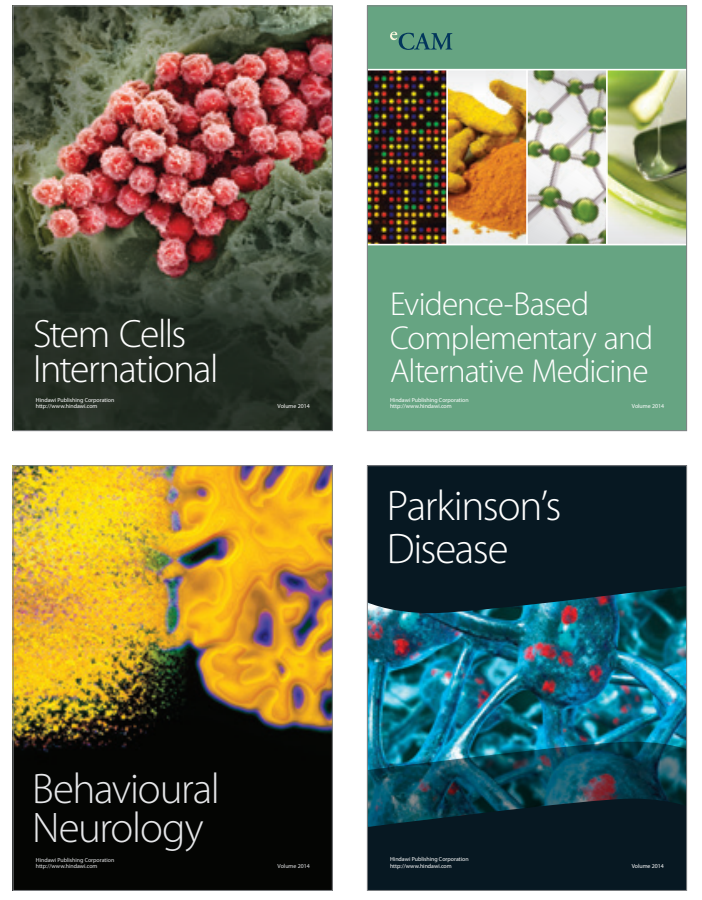
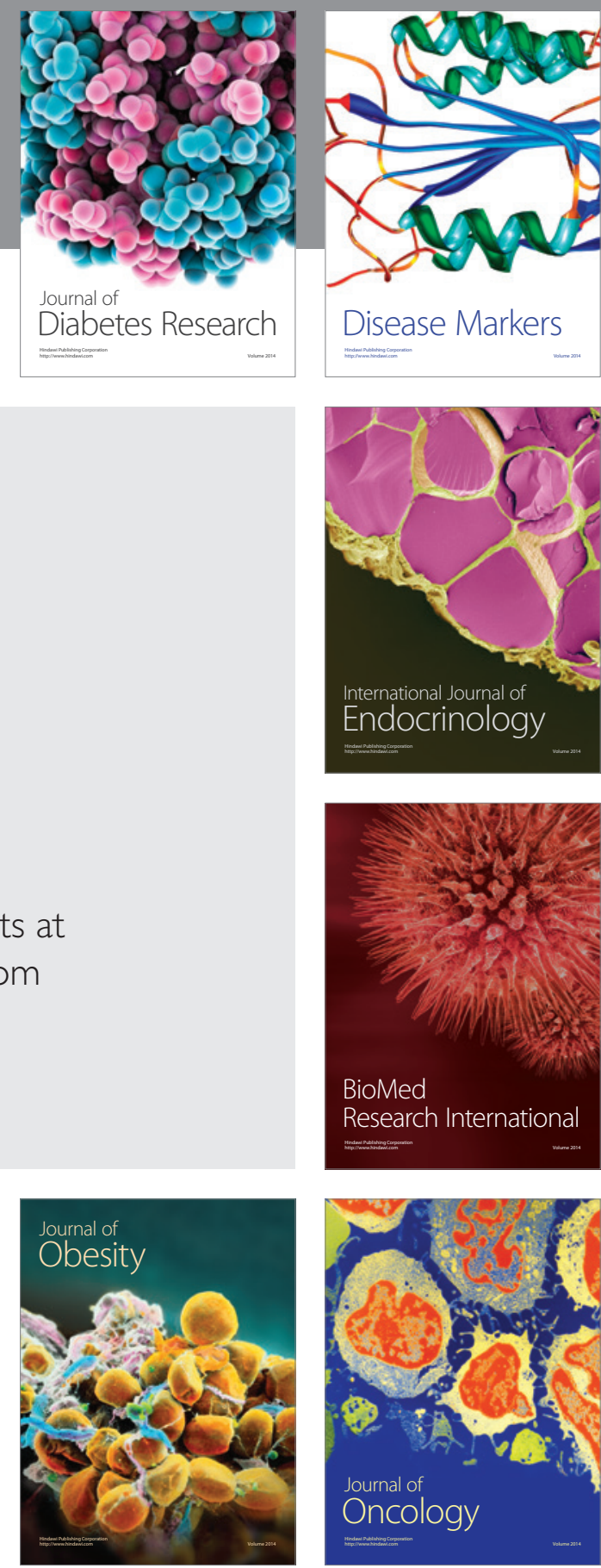

Disease Markers
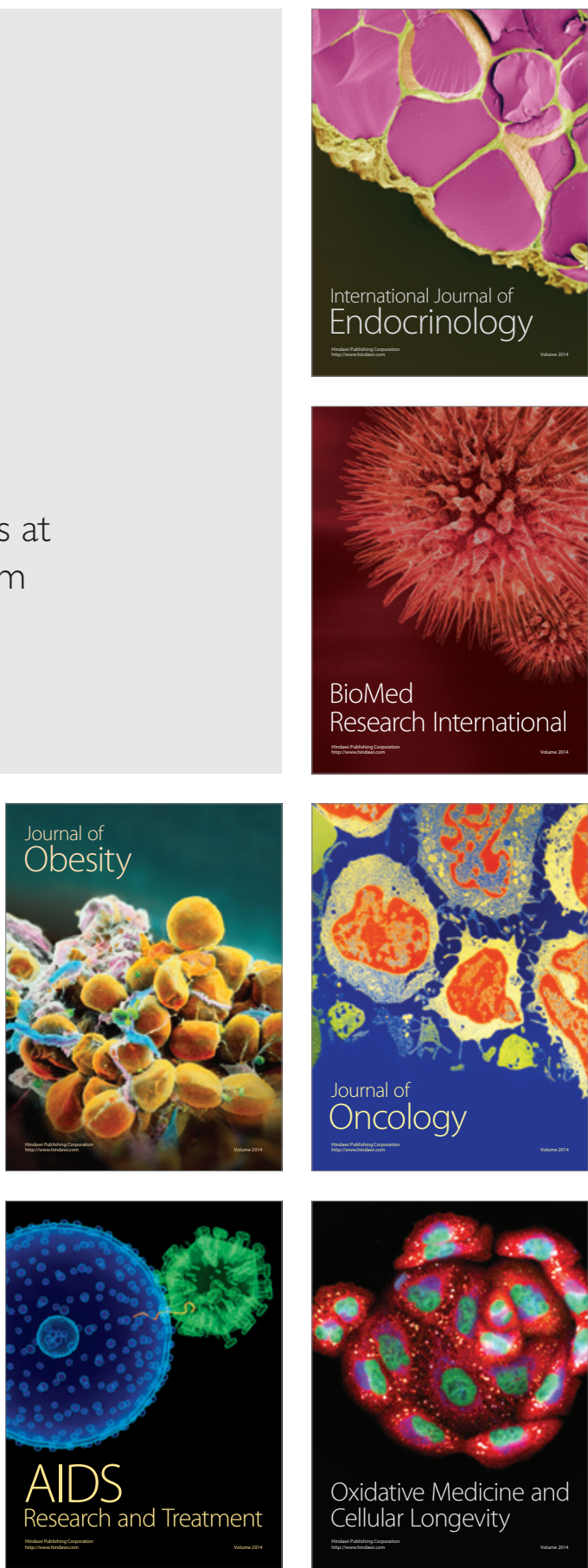